\title{
Contribuição farmacêutica na promoção da saúde em farmácias e drogarias
}

\author{
Pharmaceutical contribution to health promotion in pharmacies and drugstores \\ Contribución farmacéutica a la promoción de la salud en farmacias y droguerías
}

Recebido: 06/03/2021 | Revisado: 12/03/2021 | Aceito: 14/03/2021 | Publicado: 21/03/2021

\author{
Geise Raquel Sousa Pinto \\ ORCID: https://orcid.org/0000-0002-6444-7800 \\ Faculdade Pitágoras, Brasil \\ E-mail: geise.raquel@hotmail.com \\ Maria Madalena Côrrea Melo \\ ORCID: https://orcid.org/0000-0001-8459-6849 \\ Faculdade Pitágoras, Brasil \\ E-mail: madalena_98@outlook.com \\ Vanessa Guimarães Leal \\ ORCID: https://orcid.org/0000-0002-9579-8582 \\ Faculdade Pitágoras, Brasil \\ E-mail: vanessa.leal02@hotmail.com \\ Juliana da Silva da Costa \\ ORCID: https://orcid.org/0000-0002-4168-3421 \\ Faculdade Pitágoras, Brasil \\ E-mail: julianahb4.16@gmail.com \\ Luciana Sena Dias \\ ORCID: https://orcid.org/0000-0003-3833-8413 \\ Faculdade Pitágoras, Brasil \\ E-mail: lucianasenadias@hotmail.com \\ Camila Vitória Pinto Teixeira \\ ORCID: https://orcid.org/0000-0001-5795-5236 \\ Faculdade Pitágoras, Brasil \\ E-mail: camilateixeira096@outlook.com
}

\begin{abstract}
Resumo
Os medicamentos têm-se transformado em elementos de primeira ordem e constituem-se em ferramentas poderosas para aliviar o sofrimento humano, entretanto também produzem efeitos não desejados, sendo necessário avaliar as consequências de seu uso sob essa perspectiva. No entanto, o seu uso irracional, tem contribuído para o surgimento de muitos eventos adversos, com elevado impacto negativo sobre a saúde da população. O objetivo do trabalho foi destacar a importância do atendimento farmacêutico nas farmácias e drogarias. A metodologia adotada para a confecção foi uma revisão bibliográfica, com Foram utilizados para a pesquisa artigos, livros e dissertações da área da hematologia e farmacologia, encontradas nas bases de dados eletrônicos: PubMed, Scientific Electronic Library Online (SCIELO), Google Acadêmico, Portal de Teses da USP e Biblioteca Virtual de Saúde (BVS). O profissional farmacêutico possui ferramentas como a assistência farmacêutica, atenção farmacêutica e a intervenção farmacêutica que o possibilita realizar seu trabalho ativo junto a sociedade de forma que o paciente esteja sempre o principal beneficiário. O uso irracional de medicamentos é um importante problema de saúde pública; portanto, é preciso considerar o potencial de contribuição do farmacêutico e efetivamente incorporá-lo às equipes de saúde a fim de que se garanta a melhoria da utilização dos medicamentos.
\end{abstract}

Palavras-chave: Atenção farmacêutica; Uso racional de medicamentos; Farmácia clinica.

\begin{abstract}
Medicines have become first-rate elements and are powerful tools to alleviate human suffering, however they also produce unwanted effects, and it is necessary to evaluate the consequences of their use from this perspective. However, its irrational use has contributed to the emergence of many adverse events, with a high negative impact on the health of the population. The objective of the work was to highlight the importance of pharmaceutical services in pharmacies and drugstores. The methodology adopted for the preparation was a bibliographic review, with articles, books and dissertations in the area of hematology and pharmacology, found in the electronic databases: PubMed, Scientific Electronic Library Online (SCIELO), Google Scholar, Portal USP Theses and Virtual Health Library (VHL). The pharmaceutical professional has tools such as pharmaceutical assistance, pharmaceutical care and pharmaceutical intervention that allows him to carry out his active work with society so that the patient is always the main beneficiary. The irrational use of medications is an important public health problem; therefore, it is necessary to consider the potential contribution of the pharmacist and effectively incorporate it into the health teams in order to guarantee an improvement in the use of medications.
\end{abstract}


Keywords: Pharmaceutical attention; Rational use of medicines; Clinical pharmacy.

\section{Resumen}

Los medicamentos se han convertido en elementos de primer orden y son herramientas poderosas para aliviar el sufrimiento humano, sin embargo también producen efectos no deseados, y es necesario evaluar las consecuencias de su uso desde esta perspectiva. Sin embargo, su uso irracional ha contribuido a la aparición de muchos eventos adversos, con un alto impacto negativo en la salud de la población. El objetivo del trabajo fue resaltar la importancia de la atención farmacéutica en farmacias y droguerías. La metodología adoptada para la elaboración fue una revisión bibliográfica, con artículos, libros y disertaciones en el área de hematología y farmacología, que se encuentran en las bases de datos electrónicas: PubMed, Scientific Electronic Library Online (SCIELO), Google Scholar, Portal USP Theses y Biblioteca Virtual en Salud (BVS). El profesional farmacéutico cuenta con herramientas como la asistencia farmacéutica, la atención farmacéutica y la intervención farmacéutica que le permiten realizar su trabajo activo con la sociedad para que el paciente sea siempre el principal beneficiario. El uso irracional de medicamentos es un importante problema de salud pública; por tanto, es necesario considerar el aporte potencial del farmacéutico e incorporarlo eficazmente a los equipos de salud para garantizar una mejora en el uso de los medicamentos.

Palabras clave: Atención farmacêutica; Uso racional de medicamentos; Farmacia Clínica.

\section{Introdução}

De acordo com Brasil (2012), existem dentro das áreas das farmácias e drogarias serviços de suma importância que podem ser realizados durante o atendimento farmacêutico. Entre todas as atribuições que cabem ao profissional farmacêutico, integra-se a assistência farmacêutica que está voltada para promoção, proteção e recuperação da saúde.

Este conjunto de atividades envolve um ciclo que vai da seleção dos medicamentos, programação, aquisição, distribuição, até a sua dispensação. Porém, nos dias de hoje, a assistência farmacêutica se constitui de um desafio para a saúde pública devido à escassez de recursos (Bruns; Luiza; Oliveira, 2014).

Este trabalho proporcionou uma melhor compreensão em relação ao tema e como o profissional farmacêutico pode contribuir promovendo o uso racional de medicamento. O estudo destaca a importância do acompanhamento farmacêutico dentro das drogarias e farmácia no Brasil, bem como os serviços farmacêuticos oferecidos como: aferição de pressão arterial, teste de glicemia capilar, orientação farmacêutica e entrega de folders, visando com que essas ações reduzam os riscos do uso de medicamentos, menores índices de morbimortalidade, que está associada aos mesmos (Correr; Otuki, 2013). Diante do exposto acima, a pergunta norteadora desse trabalho foi: Como o farmacêutico pode interferir positivamente na vida dos pacientes dentro das farmácias?

Sendo assim, o presente trabalho teve como objetivo geral abordar a importância da atenção farmacêutica dentro das farmácias e drogarias. E como objetivos específicos: ressaltar as atribuições do profissional farmacêutico nas farmácias; apontar a importância do acompanhamento farmacoterapêutico e discutir a importância do farmacêutico frente ao uso racional de medicamentos nas farmácias e drogarias.

\section{Metodologia}

Trata-se de um estudo de revisão bibliográfica de cunho descritivo e com abordagem quantitativa. A pesquisa foi realizada em bases de dados Scientific Eletronic Library Online- SciELO, LILACS, Pubmed, Portal de Teses da USP, Biblioteca Virtual de Saúde (BVS) e Google Acadêmico.

Utilizaram-se descritores retirados da base de Descritores em Ciência da Saúde (DeCS), sem cruzamentos entre eles, sendo assim, aplicaram-se os descritores: atenção farmacêutica, uso racional de medicamentos, farmacêutico clínico. $\mathrm{O}$ estudo teve como critérios de inclusão, artigos publicados em periódicos internacionais e nacionais, nos idiomas inglês e português, publicados no período de janeiro de 2010 à agosto de 2021. E todos aqueles que não se enquadraram nesses critérios, foram excluídos. Foram encontrados 220 artigos no total, dentre eles 20 foram selecionados de acordo com título e resumo. O Quadro 1 a seguir mostra os artigos encontrados nas bases. 
Quadro 1. Número de artigos utilizados segundo a base de dados.

\begin{tabular}{|c|c|}
\hline Base de dados & Número de artigos \\
\hline Scielo & 5 \\
\hline LILACS & 4 \\
\hline PubMed & 2 \\
\hline Portal de Teses da USP & 2 \\
\hline BVS & 6 \\
\hline Google Acadêmico & 20 \\
\hline Total & \\
\hline
\end{tabular}

Fonte: Autores (2020).

Para a identificação dos trabalhos de interesse para estudos foi realizada a leitura do título e dos resumos dos artigos encontrados, sendo este o critério de elegibilidade. Foram desconsiderados todos aqueles que não contemplavam o tema.

\section{Resultados e Discussão}

Uma pesquisa realizada no Hospital das Clínicas da Universidade Federal do Paraná (HC-UFPR), analisou 6.438 prescrições médicas, nas quais dessas foram identificados em maior percentual os erros de dose (46,73\%), e em meio aos diferentes de PRM encontrados, como pode ser observado na Tabela 1 (Reis, 2013).

Tabela 1. Problemas relacionados ao uso de medicamentos.

\begin{tabular}{|c|c|}
\hline PRM & n (\%) \\
\hline Dose & $436(46,73)$ \\
\hline Intervalo de administração & $27(2,89)$ \\
\hline Via de administração & $1(0,11)$ \\
\hline Apresentação e/ou forma farmacêutica & $64(6,86)$ \\
\hline Medicamento inapropriado/desnecessário & $178(19,08)$ \\
\hline Necessidade de medicação adicional & $49(5,25)$ \\
\hline Alternativa terapêutica mais adequada/disponível & $73(7,82)$ \\
\hline Interação medicamentosa & $70(7,50)$ \\
\hline Inconsistência de prescrição & $31(3,32)$ \\
\hline Diluição e/u taxa de infusão & $1(0,11)$ \\
\hline $\begin{array}{l}\text { Incompatibilidades } \\
\text { estabilidade da preparação }\end{array}$ & $3(0,32)$ \\
\hline TOTAL & 933 \\
\hline
\end{tabular}


$\mathrm{Na}$ Tabela 2 identificou-se as necessidades relacionadas ao paciente (NRP)observadas em 54 fichas de pacientes atendidos no Serviço de Atenção Farmacêutica (SAF) da Universidade do Sul de Santa Catarina (Alano, et. Al., 2012).

Tabela 2. Frequência e classificação das NRP identificadas entre os pacientes atendidos no Serviço de Atenção Farmacêutica.

\begin{tabular}{lll}
\hline CLASSIFICAÇÃO DA NRP & $\mathbf{n}$ & (\%) \\
\hline $\begin{array}{l}\text { Informação sobre } \\
\text { a doença }\end{array}$ & 11 & 7,3 \\
$\begin{array}{l}\text { Cuidados com a } \\
\text { alimentação }\end{array}$ & 22 & 7,3 \\
\hline $\begin{array}{l}\text { Incentivo a prática de } \\
\text { exercícios físicos }\end{array}$ & 6 & 4,0 \\
\hline $\begin{array}{l}\text { Monitoramento } \\
\text { Encaminhamento a } \\
\text { profissionais de saúde }\end{array}$ & 29 & 19,2 \\
\hline Automedicação responsável & 17 & 11,3 \\
\hline Informação sobre & 6 & 4,0 \\
\hline a terapia farmacológica & 151 & 100 \\
\hline Outras terapias & 46 & 30,4 \\
\hline não farmacológicas & 14 & 9,2 \\
\hline TOTAL & & \\
\hline
\end{tabular}

Fonte: Alano, et al (2012).

Favorecer a adesão dos pacientes à farmacoterapia pelos pacientes é um dos principais desafios enfrentados pelos serviços de saúde. Os farmacêuticos das redes, em particular, apontaram o estabelecimento das relações sociais no interior das farmácias como uma das maiores dificuldades na sua atuação. Essas relações sociais assumem diversas interfaces, entre quais foram destacadas: o trabalho de convencer a equipe de balconistas e gerentes a colaborar, de modo a haver uma prática mais ética e menos comercial na farmácia; a resistência de alguns balconistas no sentido de implementar procedimentos orientados pelo farmacêutico; o fato de os clientes das farmácias confundirem sistematicamente o farmacêtico com o balconista; e o próprio exercício de estar em contato com o público, no esclarecimento de dúvidas, visto a falta ou precariedade de treinamento específico nas faculdades de farmácia, bem como a ausência, em boa parte dos estabelecimentos, de condições de infraestrutura mínimas para que tal se realize com a privacidade, o sigilo e a ética necessários. (Amarante, et. al., 2010).

Faz-se necessário atentar para o uso racionaldos medicamentos, de forma que os pacientesrecebam os medicamentos para a indicação apro-priada, nas doses, via de administração e dura-ção apropriadas; que não existam contra-indi-cações; que a probabilidade de ocorrência de re-ações adversas seja mínima; que a dispensaçãoseja correta e que haja aderência ao tratamento. Os serviços farmacêuticos de atenção primá-ria contribuem para a diminuição da internaçãoou do tempo de 
permanência no hospital, à as-sistência aos portadores de doenças crônicas, àprática de educação em saúde e, para uma intervenção terapêutica mais custo-efetiva (Correr, et. al., 2011).

Embora a legislação brasileira estabeleça que farmácias tenham assistência de técnico responsável e que este deva permanecer durante todo o horário de funcionamento do estabelecimento (Brasil, 1973), encontrou-se farmácias sem responsabilidade técnica, seja pela falta de contratação ou ausência do profissional. Essa situação, proibida por lei, demonstra que a fiscalização deve ser mais efetiva quanto à presença desses profissionais, não só devido à questão legal, mas também pelas implicações que sua ausência pode acarretar, como a falta de assistência ao paciente (Correr, et. al., 2011).

A Atenção Básica à Saúde constitui o primeiro nível de atenção à saúde. É o primeiro contato com o Sistema de Saúde. Compreende um conjunto de ações e serviços de clínica médica, pediátrica, ginecologia, obstetrícia, encaminhamentos para os demais níveis. A estratégia da organização da Atenção Básica é o PSF. A responsabilidade da oferta de serviços é da gestão municipal e o financiamento é responsabilidade dos três níveis de governo (Correr, et. al., 2011).

Entre as intervenções fundamentais para promover o uso racional de medicamentos está à prática da Atenção Farmacêutica. Esse processo é conceituado como uma atividade profissional em que o paciente é o principal beneficiário das ações do farmacêutico. Nela são incluídas atitudes, cuidados, compromissos, comportamentos, funções, conhecimentos, valores éticos, responsabilidades e habilidades do profissional farmacêutico na prestação da farmacoterapia, buscando obter resultados terapêuticos baseados nos indicadores de saúde e na qualidade de vida dos pacientes (Oenning, 2011).

Para o desenvolvimento dessa prática, são necessárias ações que, ancoradas ao tripé farmacêutico- -pacientemedicamento, possibilitem usar estratégias educativas como o aconselhamento terapêutico que irá contribuir não só para o uso correto de medicamentos, mas também propiciar benefícios individuais, institucionais e nacionais (Brasil, 2012).

O acompanhamento sistemático do tratamento medicamentoso (seguimento farmacoterapêutico) de usuários, que faz parte da definição de atenção farmacêutica estabelecida por Hepler e Strand (1990), foi citado por poucos entrevistados na sua compreensão sobre atenção farmacêutica. A grande maioria desconhecia o princípio da atenção farmacêutica e associava-a, simplesmente, ao fornecimento de atenção ao usuário, que ocorre, muitas vezes, na dispensação com o fornecimento de orientações. A capacitação dos farmacêuticos para atuarem em atenção farmacêutica e conhecerem melhor seu conceito, seus elementos e seu processo talvez possa ajudar na sua implantação (De Lima, 2017).

Sendo assim, pode-se analisar que as intervenções realizadas pelo profissional farmacêutico são capazes de desenvolver melhores resultados terapêuticos, garantindo segurança, eficácia e melhorias na farmacoterapia. Através da atenção farmacêutica, o farmacêutico promove o uso racional de medicamentos e permite as tomadas de decisões clínicas, além de interagir com outros profissionais de saúde, o que se mostra necessário para o melhor acompanhamento ao paciente no cuidado à saúde (De Lima, 2017).

\section{Conclusão}

Os medicamentos têm-se transformado em elementos de primeira ordem e constituem-se em ferramentas poderosas para aliviar o sofrimento humano, entretanto também produzem efeitos não desejados, sendo necessário avaliar as consequências de seu uso sob essa perspectiva. Embora constitua um excelente avanço a incorporação dos farmacêuticos no Nasf, poucos estudos abordam a execução dos serviços clínicos nos contextos da ESF.

Algumas limitações foram encontradas, como periódicos com publicações repetidas em mais de uma base de dados, falta de clareza em alguns resumos e escassez de estudos sobre o tema, principalmente no que se refere à atuação clínica do farmacêutico no Nasf. O farmacêutico na assistência farmacêutica vai além de dispensar medicamentos, uma vez que a população carece de medicamentos de qualidade, eficazes e de segurança comprovada, como exibe a politica nacional de medicamentos (PNM) desta forma trará muitos benefícios à equipe multidisciplinar que atua no ciclo da assistência 
farmacêutica e ao paciente, que é o foco principal. Diante da carência da população em relação a profissionais mais atuantes que buscam do URM, surge uma grande oportunidade para pratica da atenção farmacêutica, onde o farmacêutico desempenhara seu papel perante a sociedade.

Acredita-se que é necessário continuar o trabalho iniciado na elaboração do Consenso Brasileiro para divulgar e promover esse novo conceito de prática, de forma que os farmacêuticos brasileiros possam compreender os seus princípios filosóficos e aplicar na sua prática diária. E mais ainda, precisamos rever conceitos e práticas farmacêuticas incorporando essa nova filosofia em todas as atividades farmacêuticas direcionadas aos pacientes, pois todas contribuem para a melhoria das condições de saúde e de utilização dos medicamentos pela população brasileira.

\section{Referências}

Alano, G. M., Corrêa, T. D. S., \& Galato, D. (2012). Indicadores do serviço de atenção farmacêutica (SAF) da universidade do sul de Santa Catarina. Ciência \& Saúde Coletiva, 17(3), 757-764.

Amarante, L. C. M., Shoji, L. S., Beijo, L. A., Lourenço, E. B., \& Marques, L. A. M. (2010). A influência do acompanhamento farmacoterapêutico na adesão à terapia anti-hipertensiva e no grau de satisfação do paciente. Revista de Ciências Farmacêuticas Básica e Aplicada, 31(3).

Araújo, S. R., \& Junges, F. (2015). Papel do profissional farmacêutico no âmbito da assistência farmacêutica. Ciênc Saúde Coletiva.

Batista, S. D. C. M., Albuquerque, L. E. R., da Silva, N. M., \& dos Santos Medeiros, J. (2020). Polimedicação, Atenção Farmacêutica E Cuidado Farmacêutico. Journal of Biology \& Pharmacy and Agricultural Management, 16(4).

Bruns, S. D. F., Luiza, V. L., \& Oliveira, E. A. D. (2014). Gestão da assistência farmacêutica em municípios do estado da Paraíba (PB): olhando a aplicação de recursos públicos. Revista de Administração Pública, 48(3), 745-765.

Bovo, F., Wisniewski, P., \& Morskei, M. L. M. (2009). Atenção Farmacêutica: papel do farmacêutico na promoção da saúde. Biosaúde, 11(1), 43-56.

de Lima Correia, K. K., Rego, M. L. C. M. G., Júnior, M. R. B., \& Marques, R. A. (2017). Farmácia clínica: importância deste serviço no cuidado a saúde. Boletim Informativo Geum, 8(3), 7.

Chagas, I. D. S. (2014). Visão do paciente sobre a importância da assistência farmacêutica prestada em uma farmácia do município de Rio Tinto-PB no ano de 2012 .

Capucho, H. C., Carvalho, F. D., \& Cassiani, S. H. D. B. (2012). Farmacovigilância: gerenciamento de riscos da terapia medicamentosa para segurança do paciente. In Farmacovigilância: gerenciamento de riscos da terapia medicamentosa para segurança do paciente (pp. 201-201).

Correr, C. J., Otuki, M. F., \& Soler, O. (2011). Assistência farmacêutica integrada ao processo de cuidado em saúde: gestão clínica do medicamento. Revista Pan-Amazônica de Saúde, 2(3), 41-49.

Costa, K. S., Francisco, P. M. S. B., Malta, D. C., \& Barros, M. B. D. A. (2016). Fontes de obtenção de medicamentos para hipertensão e diabetes no Brasil: resultados de inquérito telefônico nas capitais brasileiras e no Distrito Federal, 2011. Cadernos de Saúde Pública, 32 , e00090014.

Gomes, C. A. P., Fonseca, A. L. D., Santos, F. J. P. D., Rosa, M. B., Machado, M. C., \& Fassy, M. D. F. (2007). A assistência farmacêutica na atenção à saúde. In A assistência farmaceutica na atenção à saúde (pp. 48-48).

Miranda, T. M. M., Petriccione, S., \& Ferracini Filho, F. T. (2012). WMB. Intervenções realizadas pelo farmacêutico clínico na unidade de primeiro atendimento. Einstein, 10(1), 74-78.

Nicoletti, M. A., \& Ito, R. K. (2018). Formação do farmacêutico: novo cenário de atuação profissional com o empoderamento de atribuições clínicas. Revista Saúde-UNG-Ser, 11(3/4), 49-62.

Oenning, D., Oliveira, B. V. D., \& Blatt, C. R. (2011). Conhecimento dos pacientes sobre os medicamentos prescritos após consulta médica e dispensação. Ciência \& Saúde Coletiva, 16, 3277-3283.

Pereira, A. S., Shitsuka, D. M., Parreira, F. J., \& Shitsuka, R. (2018). Metodologia da pesquisa científica. UFSM. https://repositorio. ufsm. br/bitstream/handle/1/15824/Lic_Computacao_MetodologiaPesquisa-Cientifica.pdf.

Reis, W. C. T., Scopel, C. T., Correr, C. J., \& Andrzejevski, V. M. S. (2013). Análise das intervenções de farmacêuticos clínicos em um hospital de ensino terciário do Brasil. Einstein (São Paulo), 11(2), 190-196.

Soler, O., Rosa, M. B., Fonseca, A. L., Fassy, M. F., Machado, M. C., Silva, R. M. C., \& Gomes, C. A. P. (2010). Assistência farmacêutica clínica na atenção primária à saúde por meio do programa saúde da família. Rev Bras Farm, 91(1), 37-45.

Sousa, J. S., de Almeida Consoline, N., Anízio, P. D., Morsch, P., \& Fagundes, D. S. (2018). A atuação da fisioterapia na prevenção de úlceras do pé diabético. Revista Científica da Faculdade de Educação e Meio Ambiente, 9(1), 320-324.

Varallo, F. R., \& Mastroianni, P. C. (2013). Farmacovigilância: avaliação do risco/benefício para a promoção do uso seguro de medicamentos. Mastroianni PC, Varallo FR, org. Farmacovigilância para promoção do uso correto de medicamentos. Artmed, 13-26. 